\title{
Search for variable stars in the young open cluster Stock 18
}

\author{
Tirthendu Sinha ${ }^{1, \star}$, Saurabh Sharma ${ }^{1, \star \star}$, Rakesh Pandey ${ }^{1}$, and A. K. Pandey ${ }^{1}$ \\ ${ }^{1}$ Aryabhatta Research Institute of Observational Sciences (ARIES), Manora Peak, Nainital, 263 001, India
}

\begin{abstract}
We have carried out multi-epoch deep $I$ band photometry of the open cluster Stock 18 to search for variable stars in star forming regions. In the present study, we report identification of 28 periodic and 165 non-periodic variables stars. The periods of most of the periodic variables are in between 2 to 20 hours and their magnitude varies between 0.05 to $0.6 \mathrm{mag}$. We have derived spectral energy distributions for 48 probable pre-main sequence variables and the average age and mass is found to be $2.7 \pm 0.3 \mathrm{Myrs}$ and $2.7 \pm 0.2 \mathrm{M}_{\odot}$, respectively.
\end{abstract}

\section{Introduction}

Young star clusters are potential sites for studying the characteristics of pre-main sequence (PMS) stars as they are found in plenty of numbers in these regions and they have broad mass range as well as spreads (few Myrs) in their ages. These stars have circumstellar disks around them during their early evolution. The PMS stars usually show a wide range of variability in their luminosity in almost all wavelengths from X-ray to infrared, and the variability time scale ranges from few minutes to years ([1]). Photometric variability results from several facts like irregular distribution of cool spots on stellar photosphere, variable hot spots, obscuration from dust ([3], and reference therein), instability in disk, change in accretion rate, etc. Due to the presence of cool and hot spots on the photosphere, the rotation of the star will give periodic/quasi-periodic variability. The evolution of disks and the accretion rates may also play a prominent role in non-periodic variability ([4-6]). The young open cluster (age $\sim 6 \mathrm{Myr}$, [2]) Stock $18(\mathrm{RA}=00: 01: 37, \mathrm{DEC}=+64: 37: 30)$ is an ideal site to search for PMS variable stars and to study their properties.

\section{Observation and data analysis}

Optical photometric observations of Stock 18 were taken on the nights of 31/09, 21/10, 11/11, 26/11, $17 / 12$ and 26/12 of year 2016 using the $1.3 \mathrm{~m} \mathrm{f} / 4$ Devasthal telescope. The telescope has a $2048 \times 2048$ pixel square CCD camera covering $18.4 \times 18.4 \mathrm{arcmin}^{2}$ field of view. In total, about 325 and 13 frames were taken in $I$ and $V$ filters, respectively, with exposure of $180 \mathrm{sec}$ each. The basic image processing such as bias subtraction and flat fielding was done by using tasks available within IRAF. Instrumental magnitude was obtained by PSF fitting using the DAOPHOT package. We have used the DAOMATCH and DAOMASTER routine of DAOPHOT-II to estimate the shifts in the individual

$\star$ tirthendu@aries.res.in

$\star \star$ saurabh@aries.res.in 

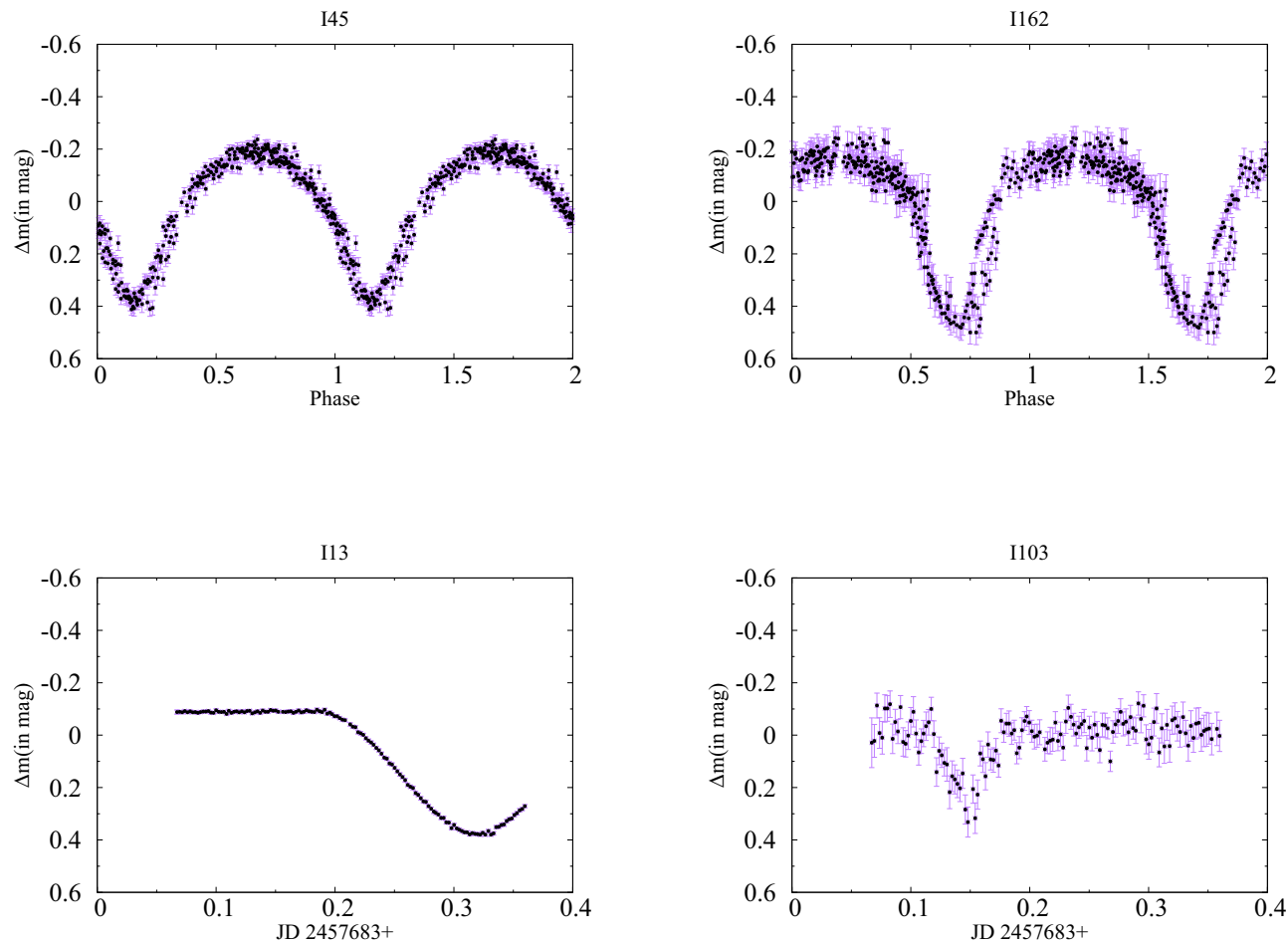

Figure 1. First two panels: Phase folded LC for periodic variables, last two panels: LC for non-periodic variables.

frames with respect to a reference frame and to get magnitudes of stars detected in different frames. Calibration of instrumental magnitude to the standard system was done by using the photometric data published by [2].

\section{Result and conclusion}

We have calculated the standard deviation (SD) of the difference of magnitude of all the stars in various magnitude bins (e.g., 10-11, 11-12, 12-13, and so on) and the stars in each magnitude bin having smallest SD are taken as comparison stars for that bin. The differential magnitude $(\Delta m)$ in the sense star - comparison were plotted as a function of Julian date. The probable variables were identified by visual inspection. To determine the probable period of the variable stars we used Period software. We have identified 28 periodic and 165 non periodic variables in the region up to a $V$ band magnitude of 20 mag. Light curves (LCs) of a few variables are shown in Figure 1. Most of the periodic variables have periods ranging from $2-20$ hours and their magnitude varies between $0.05-0.6 \mathrm{mag}$. 
The evolutionary status of these variables was explored through their position in the HertzsprungRussell diagram (for details, see [7]). Out of the detected variables, 168 have good photometric data (error $<0.1$ ) and 80 of them have ages $>10$ Myr. They are most probably main sequence or field stars. The average age of the remaining variables is found to be $4.2 \pm 0.4$ Myrs (88 stars). We have also derived the spectral energy distribution (SED) for the probable PMS-stars (having ages $<4.6 \mathrm{Myr}$, i.e., average age + standard error) using the available optical, near-infrared (2MASS) and mid-infrared (WISE) data, with a condition that they have photometric data in at least 5 broad band filters. We derived SEDs for 48 probable PMS variables (5 of them are also showing infrared excess). From the SED fitting analyses (for details, cf. [7]), the average age and mass of probable PMS-variables come out to be $2.7 \pm 0.3$ Myrs and $2.7 \pm 0.2 \mathrm{M}_{\odot}$, respectively which suggests that these stars should be T Tauri stars. Disk/envelope accretion rates and masses of these PMS variable stars are also determined from the SED fitting and detailed analyses is underway.

\section{References}

[1] Appenzeller, I., \& Mundt, R., A\&ARv, 1, 291 (1989)

[2] Bhatt, H., Sagar, R., \& Pandey, J. C., New Astronomy, 17, 160 (2012)

[3] Herbst, W., Herbst, D. K., Grossman, E. J., \& Weinstein, D., AJ, 108, 1906 (1994)

[4] Hillenbrand, L. A, preprint (arXiv : astro-ph/0210520) (2002)

[5] Percy, J. R., Gryc, W. K., Wong, J. C.-Y., \& Herbst, W., PASP, 118, 1390 (2006)

[6] Percy, J. R., Grynko, S., Seneviratne, R., \& Herbst, W., PASP, 122, 753 (2010)

[7] Sharma, S., Pandey, A. K., Ojha, D. K., et al., MNRAS, 467, 2943 (2017) 